\title{
Association of familial macular degeneration with specific genetic markers: a case report
}

\author{
Yoshinori Takayanagi ${ }^{1,2^{*}}$, Masami Ashida², Mayumi Go², Mai Gunji², Izuru Sato', Shigeaki Kato ${ }^{2,3^{*}}$ \\ and Masato Miyashita, ${ }^{1,2}$
}

\begin{abstract}
Introduction: Age-related macular degeneration is a serious visual disorder of the central retina and was recently reported to be associated with genetic background. Here we describe a genetic link to early onset age-related macular degeneration in members of an Asian family.

Case presentation: A 73-year-old Asian woman developed age-related macular degeneration in the fifth decade of her life and her 49-year-old daughter developed age-related macular degeneration. Because of the family history and the early onset, family members were tested for two single nucleotide polymorphism variants (rs10490924 and rs11200638) at a recently identified susceptibility locus for age-related macular degeneration. Both alleles in the 73-year-old woman were of the high-risk variants (T/T for rs10490924 and A/A for rs11200638), and her two daughters and a grandson each carried the risk variants ( $T$ and $A$ ) one on each allele.

Conclusions: In a case where multiple family members had early onset age-related macular degeneration, we found two high-risk single nucleotide polymorphism variants in the age-related macular degeneration susceptibility locus, suggesting the combination of the known single nucleotide polymorphism variants as a potent age-related macular degeneration diagnostic indicator.
\end{abstract}

Keywords: Age-related macular degeneration, ARMS2 gene, Susceptibility gene locus

\section{Introduction}

Age-related macular degeneration (AMD) is a serious visual disorder of the central retina and it is prevalent in developed countries [1, 2]. In Asian patients, exudative (wet) AMD occurs more frequently than geographic atrophy (dry) AMD seen in European patients with late AMD $[3,4]$. Choroidal vasculopathy around the macula triggers abnormal neovascularization, leading to serious hemorrhaging and exudation [5]. For most European patients, without such hemorrhages, retinal thinning is accompanied by atrophy of the retinal pigment epithelium [5].

\footnotetext{
* Correspondence: takayanagi@carnamed.jp; uskato0525@gmail.com ${ }^{1}$ CARNAMED Eye Clinic, Sapporo S1 Building 3F, Nishi4-20-5, Minami1-jo, Chuouku, Sapporo, Hokkaido 060-0807, Japan

2DAL-DNA Analysis Laboratory, Co. Ltd, Sapporo North, Building 3F, Nishi2-8-1, Kita7-jo, Kitaku, Sapporo, Hokkaido 060-0807, Japan Full list of author information is available at the end of the article
}

Irrespective of similarities in phenotypic defects among AMD types, the mechanisms underlying the development of $\mathrm{AMD}$ are not well understood. However, from the familial incidence of AMD, genetic background has been suspected and, accordingly, genes associated with AMD have been identified for both the dry and wet types of AMD [6-8]. Recent genetic association studies have been successful in identifying several AMD riskassociated single nucleotide polymorphism (SNP) variants, and the SNP variants are specific to AMD type, reflecting different genetic backgrounds among races. Through genome-wide association studies (GWASs) on large populations of patients with AMD that included independent groups, several SNP susceptibility variants were identified in genes encoding complement factor $\mathrm{H}$ $(C F H)$, high-temperature requirement factor A1 (HTRA1) and age-related maculopathy susceptibility 2 (ARMS2), as well as in the regions for TNFSF10A- 
LOC389641 and REST-C4orf14-POLR2B-IGFBP7 for dry-type [9] and for wet-type AMD [10, 11].

Diagnosis of AMD is certified for patients over 50 years of age, but there is also a group of patients with AMD with early onset $[1,2]$. For such patients, hereditary SNP variation in loci associated with AMD is assumed. We report here an Asian case of familial AMD with early onset bearing both of the known high-risk SNP variants (rs10490924 and rs11200638 in ARMS2 and HTRA1, respectively) $[10,11]$ in patients with AMD, suggesting a genetic association between the two SNPs in AMD onset.

\section{Case presentation}

A 73-year-old Asian woman consulted 19 years ago (1996) requesting examination of her right eye, since both eyes bore pterygium and her left eye was diagnosed as having wet-type AMD in another ophthalmological clinic 23 years ago (1992); she is designated as Patient A (PA) in the rest of this case report. She was reported to have blurriness in her central visual field in the early part of the third decade of her life. Intraocular pressure was $14 \mathrm{mmHg}$ in her right eye and $12 \mathrm{mmHg}$ in her left eye, and no overt abnormalities were seen in the cornea or the crystalline lens in either eye by slit lamp test in 1996. A fluorescein angiogram (FAG) in 1996 and a fundus examination in 2007 of her right eye showed classical wet-type AMD with choroidal neovascularization, hyperpigmentation and reticular pseudodrusen (Fig. 1). Her best-corrected visual acuity was 20/25 for her right eye and 20/30 for her left eye in 1992, but these acuities gradually decreased over several years to $20 / 300$ for her left eye and, in 2003, they reached less than 20/ 400 in both eyes. A SNP survey was conducted last year for the recently reported susceptibility loci ARMS2 and HTRA1 for wet AMD (Fig. 2a). Both SNP variants in this patient (PA) were high-risk alleles: $\mathrm{T} / \mathrm{T}$ for rs10490924 in the coding region (a mutation that converts alanine 69 into serine in the ARMS2 protein; A69S) and A/A for rs11200638 in the promoter of the HTRA1 gene (Fig. 2a, b) [10, 11]. After these findings from SNP analysis, one of PA's daughters, who was 49 years of age (Patient B; PB), visited our hospital for a SNP survey; she also manifested blurred central vision in her left eye and was afraid of AMD. Her best-corrected visual acuity was 20/20 for her right eye and 20/25 for her left eye. Intraocular pressure was $16 \mathrm{mmHg}$ for her right eye and $15 \mathrm{mmHg}$ for her left eye; neither eye exhibited overt abnormalities by slit lamp examination. However, in a fundus examination, abnormality of the retinal pigment epithelium was detected, as well as reticular pseudodrusen, and she was diagnosed as having classic wet AMD in 2014 (Fig. 1). To prevent AMD progression, an anti-vascular endothelial growth factor (VEGF) antibody, Eylea (aflibercept ophthalmic), for the left eye of PB was administered in 2014. After 10 months, she reported that blurring was ameliorated and the best-corrected visual acuity for her left eye was consistently found to be $20 / 20$. PB and her younger sister, at 45 years of age (Patient C; PC), took the SNP survey (Fig. 2b, c). High-risk variants were found in both high-risk loci: $\mathrm{T}$ for rs10490924 and A for rs11200638 [11, 12]. The younger sister

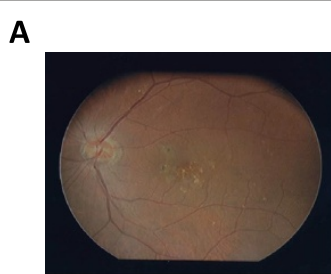

PA ( Left eye)

B

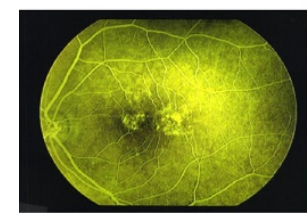

PA ( Left eye)

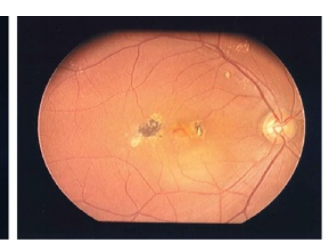

PA ( Right eye)

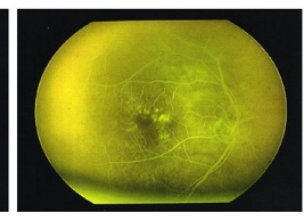

PA ( Right eye)

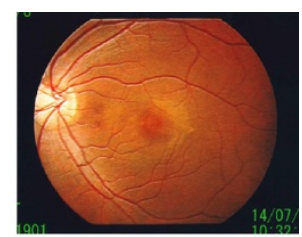

PB ( Left eye)

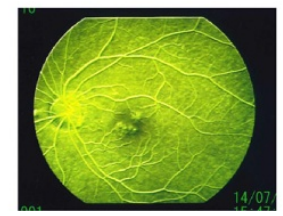

PB ( Left eye)

Fig. 1 Color fundus photographs and fluorescein angiograms. a Fundus photographs of the patients (PA and PB are the family members depicted in Fig. 2). The images for PA were taken in 2007 and those for PB in 2014. b Fluorescein angiograms for PA taken in 1996 and PB in 2014. Choroidal neovascularization, hyperpigmentation and reticular pseudodrusen were seen as representative markers for classical wet type of age-related macular degeneration. PA Patient A, PB Patient B 


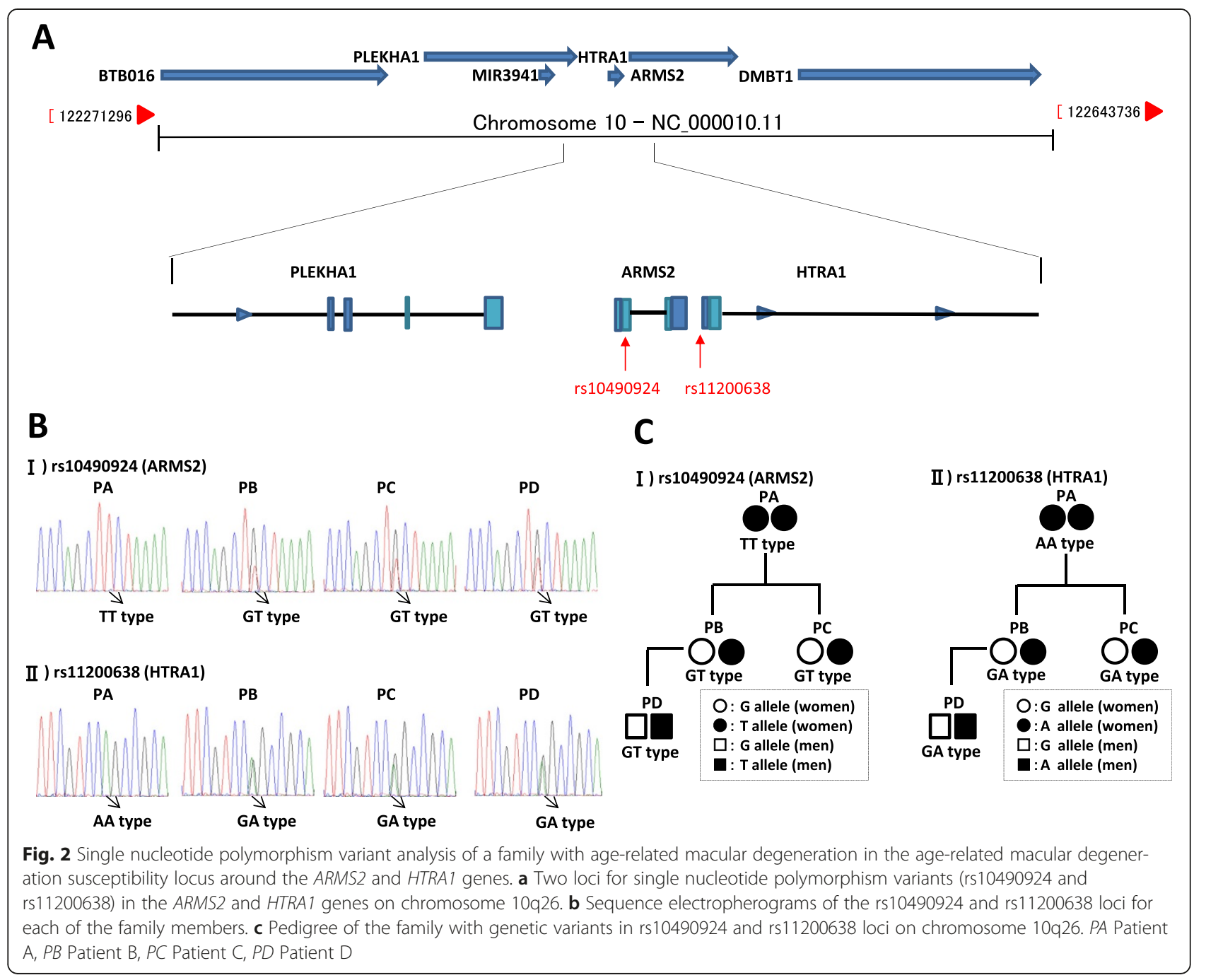

(PC) and the grandson (Patient D; PD) had no manifestations of any AMD-related visual defect.

\section{Discussion}

AMD is a common eye condition among patients aged 50 years and older [1,2]. The onset and progression of AMD are diverse, but AMD, per se, is generally not serious enough to cause complete blindness [1, 2]. However, the loss of central vision from damage to the macula threatens the visual abilities that support every aspect of normal life, since the center of the field of view is distorted [1-4].

Although common features are detected among patients with AMD, the molecular basis of AMD onset and development remains to be uncovered. Moreover, effective treatments and drugs to prevent AMD are still in clinical trials $[12,13]$. In this respect, investigation of genetic variants in patients with AMD in large populations is a promising strategy for identifying molecular targets for intervention in AMD, as it is well established that familial history is a strong predictor of AMD. Among the AMD susceptibility loci [6-11], we identified two SNP variants that mutated A69 of the ARMS2 protein into alanine (A69S) (T/T for rs10490924) and a variation with high risk (A/A) in the promoter of the HTRA1 gene at rs11200638, since these SNP variants are predictive of AMD incidence with high odds ratios (2.86) by GWAS analyses of large numbers of patients with AMD [10]. Moreover, a mechanistic link between the ARMS2 A69S mutation and AMD progression has been illustrated [10]. Both of the tested high-risk alleles ( $\mathrm{T}$ for rs10490924 and A for rs11200638) were found in all of the Asian family members, and all of them had had dry eye since adolescence. As the relationship among the multiple susceptibility gene loci delineated by several independent groups remains to be studied in terms of development and onset of AMD, our findings are intriguing because they suggest mutual associations among the identified susceptibility gene loci. Further assessment of other SNP variants related to AMD may 
add more genetic information. This study has many limitations because it is a case report and needs more examination to assess our conclusions. It is also evident that other factors underlie the onset and development of AMD. However, the present case study represents a rational approach to provide a prevention strategy for those at risk for AMD, particularly for those with a familial AMD history.

\section{Conclusions}

An Asian family with early onset AMD bore the known high-risk SNP variants in both ARMS2 and HTRA1; the presence of both SNPs could be an indicator for early onset AMD.

\section{Consent}

Written informed consent was obtained from the patients for publication of this case report and any accompanying images. Copies of written consents are available for review by the Editor-in-Chief of this journal.

\section{Abbreviations}

AMD: Age-related macular degeneration; ARMS2: Age-related maculopathy susceptibility 2; GWAS: Genome-wide association study; HTRA1: Hightemperature requirement factor A1; PA: Patient A; PB: Patient B; PC: Patient C; SNP: Single nucleotide polymorphism.

\section{Competing interests}

The authors declare that they have no competing interests.

\section{Authors' contributions}

YT carried out clinical examinations and prepared the images of the patients. MA, MG and MG conducted genetic assays and prepared figures. YT, IS and SK designed the study and SK drafted the manuscript. All authors read and approved the final manuscript.

\section{Acknowledgement}

We thank Dr Shiho Kunimatsu-Sanuki (Tohoku University Graduate School of Medicine) for critical reading and valuable comments to complete the study.

\section{Author details}

${ }^{1}$ CARNAMED Eye Clinic, Sapporo S1 Building 3F, Nishi4-20-5, Minami1-jo, Chuouku, Sapporo, Hokkaido 060-0807, Japan. ${ }^{2}$ DAL-DNA Analysis Laboratory, Co. Ltd, Sapporo North, Building 3F, Nishi2-8-1, Kita7-jo, Kitaku, Sapporo, Hokkaido 060-0807, Japan. ${ }^{3}$ Research Center, Jyoban Hospital, 57 Kaminodai, Jyoban, Kamiyunagayamachi, Iwaki, Fukushima 972-8322, Japan.

Received: 10 April 2015 Accepted: 9 November 2015

Published online: 24 November 2015

\section{References}

1. Wong TY, Chakravarthy U, Klein R, Mitchell P, Zlateva G, Buggage R, et al. The natural history and prognosis of neovascular age-related macular degeneration: a systematic review of the literature and meta-analysis. Ophthalmology. 2008;115(1):116-26.

2. Smith W, Assink J, Klein R, Mitchell P, Klaver CC, Klein BE, et al. Risk factors for age-related macular degeneration: Pooled findings from three continents. Ophthalmology. 2001;108(4):697-704.

3. Kawasaki R, Yasuda M, Song SJ, Chen SJ, Jonas JB, Wang JJ, et al. The prevalence of age-related macular degeneration in Asians: a systematic review and meta-analysis. Ophthalmology. 2010;117(5):921-7.

4. Laude A, Cackett PD, Vithana EN, Yeo IY, Wong D, Koh AH, et al. Polypoidal choroidal vasculopathy and neovascular age-related macular degeneration: same or different disease. Prog Retin Eye Res. 2010;29(1):19-29.
5. Ding $X$, Patel M, Chan CC. Molecular pathology of age-related macular degeneration. Prog Retin Eye Res. 2009;28(1):1-18.

6. Klein RJ, Zeiss C, Chew EY, Tsai JY, Sackler RS, Haynes C, et al. Complement factor $\mathrm{H}$ polymorphism in age-related macular degeneration. Science. 2005; 308(5720):385-9.

7. Yang Z, Camp NJ, Sun H, Tong Z, Gibbs D, Cameron DJ, et al. A variant of the HTRA1 gene increases susceptibility to age-related macular degeneration. Science. 2006;314(5801):992-3.

8. Dewan A, Liu M, Hartman S, Zhang SS, Liu DT, Zhao C, et al. HTRA1 promoter polymorphism in wet age-related macular degeneration. Science. 2006;314(5801):989-92.

9. Fritsche $L G$, Loenhardt $T$, Janssen A, Fisher SA, Rivera A, Keilhauer CN, et al. Age-related macular degeneration is associated with an unstable ARMS2 (LOC387715) mRNA. Nat Genet. 2008;40(7):892-6.

10. Arakawa S, Takahashi A, Ashikawa K, Hosono N, Aoi T, Yasuda M, et al. Genome-wide association study identifies two susceptibility loci for exudative age-related macular degeneration in the Japanese population. Nat Genet. 2011;43(10):1001-4.

11. Tamura H, Tsujikawa A, Yamashiro K, Akagi-Kurashige Y, Nakata I, Nakanishi $\mathrm{H}$, et al. Association of ARMS2 genotype with bilateral involvement of exudative age-related macular degeneration. Am J Ophthalmol. 2012;154(3): 542-8.

12. Cheung $C M$, Wong TY. Is age-related macular degeneration a manifestation of systemic disease. New prospects for early intervention and treatment. J Intern Med. 2014;276(2):140-53.

13. Glanville J, Patterson J, McCool R, Ferreira A, Gairy K, Pearce I. Efficacy and safety of widely used treatments for macular oedema secondary to retinal vein occlusion: a systematic review. BMC Ophthalmol. 2014;14:7.

\section{Submit your next manuscript to BioMed Central and take full advantage of:}

- Convenient online submission

- Thorough peer review

- No space constraints or color figure charges

- Immediate publication on acceptance

- Inclusion in PubMed, CAS, Scopus and Google Scholar

- Research which is freely available for redistribution 